\title{
Inventario de hábitats y flora amenazada de la ZEC Jaizkibel
}

\author{
Jaizkibel KBEko habitat eta flora mehatxatuen inbentarioa \\ Inventory of threatened habitats and flora in Jaizkibel SAC
}

\author{
Anaïs Mitxelena ${ }^{1 *}$, Jesús Sáenz, Leire Oreja ${ }^{1}$ \\ ${ }^{1}$ Departamento de Botánica, Sociedad de Ciencias Aranzadi, Zorroagagaina 11, 20014 Donostia-San Sebastián. \\ * Correspondencia: botanika@aranzadi.eus
}

\section{RESUMEN}

Se han actualizado la cartografía de hábitats y el inventario de la flora amenazada de la Zona de Especial Conservación Jaizkibe (ES2120017) mediante trabajo de campo realizado en 2009 y búsqueda bibliográfica y recopilación de datos realizada hasta 2013. En un área comprendida de 2470 ha, el $71 \%$ está ocupado por 26 hábitats naturales y seminaturales, de los cuales 12 son hábitats de interés comunitario. Respecto a la flora amenazada, se nombran 18 especies de las cuales no se ha podido confirmar la presencia de Carex hostiana DC desde 1953, Dryopteris carthusiana (Vill) H.P. Fuchs. desde 1967, Radiola linoides Roth. desde 1985 y Rhynchospora fusca (L.) W.T. Aiton. desde 1941

PALABRAS CLAVE: Cartografía, listado, vegetación, especies, actualización.

\section{LABURPENA}

Kontserbazio Bereziko Eremu (ES2120017) moduan izendatua dagoen Jaizkibeleko habitaten kartografia eta landare mehatxatuen inbentarioa berritu da 2009an burututako mendi-irteera eta 2013 arte jasotako datuen eta bilaketa bibliografikoez baliatuz. 2470 ha duen eremuaren \%71, 26 habitat natural eta erdinaturalez osatuta dago eta hauetatik 12 europar batasunarentzat interesekoak dira. Landare mehatxatue dagokienez, 18 espezie zerrendatu dira baina ezin izan da Carex hostiana DC. 1953. urtetik, Dryopteris carthusiana (Vill) H.P. Fuchs. 1967tik, Radiola linoides Roth. 1985tik eta Rhynchospora fusca (L.) W.T. Aiton. 1941tik espezieen presentzia ziurtatu.

GAKO-HITZAK: Kartografia, zerrenda, landaredia, espezieak, eguneratzea.

\section{ABSTRACT}

The habitat cartography has been updated as well as the inventory of the threatened flora in Jaizkibel Special Area of Conservation by means of field work undertaken in 2009 and bibliographical research and data collection undertaken until 2013. In an area comprising 2,470ha, $71 \%$ is covered by 26 natural and semi-natural habitats of which 12 are community interest habitats. Regarding the threatened flora, 18 species have been named and it has not been possible to confirm the presence of Carex hostiana DC. since 1953, Dryopteris carthusiana (Vill) H.P. Fuchs. since 1967, Radiola linoides Roth. since 1985 y Rhynchospora fusca (L.) W.T. Aiton. since 1941.

KEY WORDS: Cartography, list, vegetation, species, updating.

\section{INTRODUCCIÓN}

Durante años, el paisaje del monte Jaizkibel ha sido modelado por la intensa intervención humana. Su vegetación potencial, formada mayoritariamente por marojal, y, en menor medida, por robledal, vegetación típica de acantilados costeros y aliseda, ha sido modificada hasta conseguir como resultado el paisaje que presenta actualmente el macizo: un paisaje mucho más complejo formado por un mosaico de pequeñas manchas de bosques, grandes superficies de matorrales, prados y pastos, plantaciones forestales y acantilados costeros.

La localización de Jaizkibel, en plena costa, y su orografía han ayudado en parte a esta diversidad de hábitats y microhábitats de la que está dotado el macizo, lo que ha facilitado la presencia de endemismos y de especies vegetales poco comunes en la zona. Son de destacar, por ejemplo, las comunidades herbáceas y fruticosas y algunas especies de flora casmofítica silicícola características del medio salino y ventoso que encuentran refugio en los escabrosos acantilados litorales (OSTADAR, S.L., 2013) o algunos helechos paleotropicales que buscan cobijo en los encajonados y sombríos valles que dan al mar.
Así, dada la enorme diversidad biológica que alberga, Jaizkibel fue designado Lugar de Importancia Comunitaria (LIC) en 2004 y en 2013 ha sido declarado como Zona Especial de Conservación (ZEC), en las que se han fijado las medidas de conservación necesarias para los hábitats naturales y seminaturales y especies más interesantes (OSTADAR, S.L. 2013).

Sin embargo, ni su singularidad ni su riqueza han evitado que los estudios realizados hasta hoy sobre los hábitats y la flora del lugar hayan sido trabajos puntuales. Hoy en día no existen estudios dirigidos al conjunto del macizo de Jaizkibel y los trabajos existentes son estudios enfocados a determinados hábitats o especies que se pueden encontrar en la zona, como trabajos de gestión de turberas y esfagnales (Zendoia et al., 2003; Zendoia et al., 2006; Zendoia et al., 2007; Heras et al., 2009; Azpiroz et al., 2012; Garmendia et al., 2014) o aportaciones a catálogos florísticos con observaciones realizadas intencionadamente o al azar (Allorge \& Allorge, 1941; Braun-Blanquet, 1967; Catalán \& Aizpuru, 1985; Sanz-Azkue et al., en prensa). Por todo ello, se puede afirmar que actualmente la información dis- 
ponible sobre el lugar es escasa y se encuentra dispersa en distintas publicaciones.

El único trabajo recopilatorio resumido y actualizado existente con la información de los valores naturales del lugar, es el Documento de Objetivos y Medidas para la designación de la Zona Especial de Conservación Jaizkibel (ES2120017) (OSTADAR, S.L., 2013) sobre el que se basa en gran medida el presente trabajo.

Mediante este artículo, se pretende recopilar los datos de vegetación y flora de Jaizkibel, actualizando la cartografía detallada de los hábitats naturales y seminaturales y realizando una nueva lista de la flora amenazada del macizo.

\section{MATERIAL Y MÉTODOS}

\section{Área de estudio}

El área de estudio se centra en el Monte Jaizkibel (Gipuzkoa), más en concreto en la superficie propuesta como ZEC de Jaizkibel (ES2120017) (OSTADAR, S.L., 2013) (Fig. 1). Jaizkibel se ubica en la costa cantábrica por lo que el clima es oceánico con temperaturas suaves y precipitaciones elevadas, con valores medios anuales en torno a los $1200 \mathrm{l} / \mathrm{m}^{2}$ y los $11,6 \stackrel{\circ}{\circ}$ respectivamente (valores obtenidos en la estación de Jaizkibel entre los años 2004 y 2010, fuente Banco de Datos Eustat). En total, se calcula una superficie de 2470 ha con un rango altitudinal que va desde los $0 \mathrm{~m}$, a nivel del mar, hasta los 540 $m$. Se trata de una elevación montañosa de geomorfología compleja, orientada de suroeste (SW) a noreste (NE) en el que predomina la vertiente norte frente a la sur. Predomina el sustrato arenoso y la vertiente NE incluye areniscas y conglomerados, mientras que la vertiente SW incluye rocas algo más variadas.

\section{Revisión de la cartografía de hábitats}

La revisión y actualización de la cartografía de hábitats de Jaizkibel se efectuó en el año 2009 durante la redacción del Documento de Objetivos y Medidas para la designación de la Zona Especial de Conservación Jaizkibel (ES2120017) (OSTADAR, S.L. 2013).

Como base para dicha tarea se utilizaron el mapa de vegetación con leyenda EUNIS (European Nature Information System) y el mapa de los hábitats de interés comunitario (Anexo I de la Directiva 92/43/CEE, de Hábitats) de la Comunidad Autónoma del País Vasco (CAPV) ambos a escala 1:10.000 y elaborados por el Departamento de Medio Ambiente, Planificación Territorial, Agricultura y Pesca del Gobierno Vasco (2007)

Previo al trabajo de campo y mediante el software informático ARCGIS 9.3. se realizaron algunos cambios en la cartografía de hábitats mediante la fotointerpretación de las ortofotografías más recientes disponibles (Gobierno Vasco, 2009). Durante esta labor se insertó la cartografía detallada de los esfagnales de Jaizkibel (Zendoia et al., 2003 y 2007). Seguidamente se efectuaron salidas de campo para confirmar los cambios realizados mediante la fotointerpretación y cartografiar con el mayor detalle posible la distribución de los hábitats con el fin de actualizar la cartografía del mapa de hábitats EUNIS y por consiguiente de los hábitats de interés comunitario.

Una vez realizada la revisión cartográfica se calcularon los siguientes datos con cada hábitat natural y seminatural en Jaizkibel: número de recintos, superficie en hectáreas para cada hábitat y porcentaje que ocupa el hábitat en relación con la superficie total de Jaizkibel.

Para la discusión de los datos de hábitats naturales y seminaturales también se utilizaron el mapa de LICs ofi-

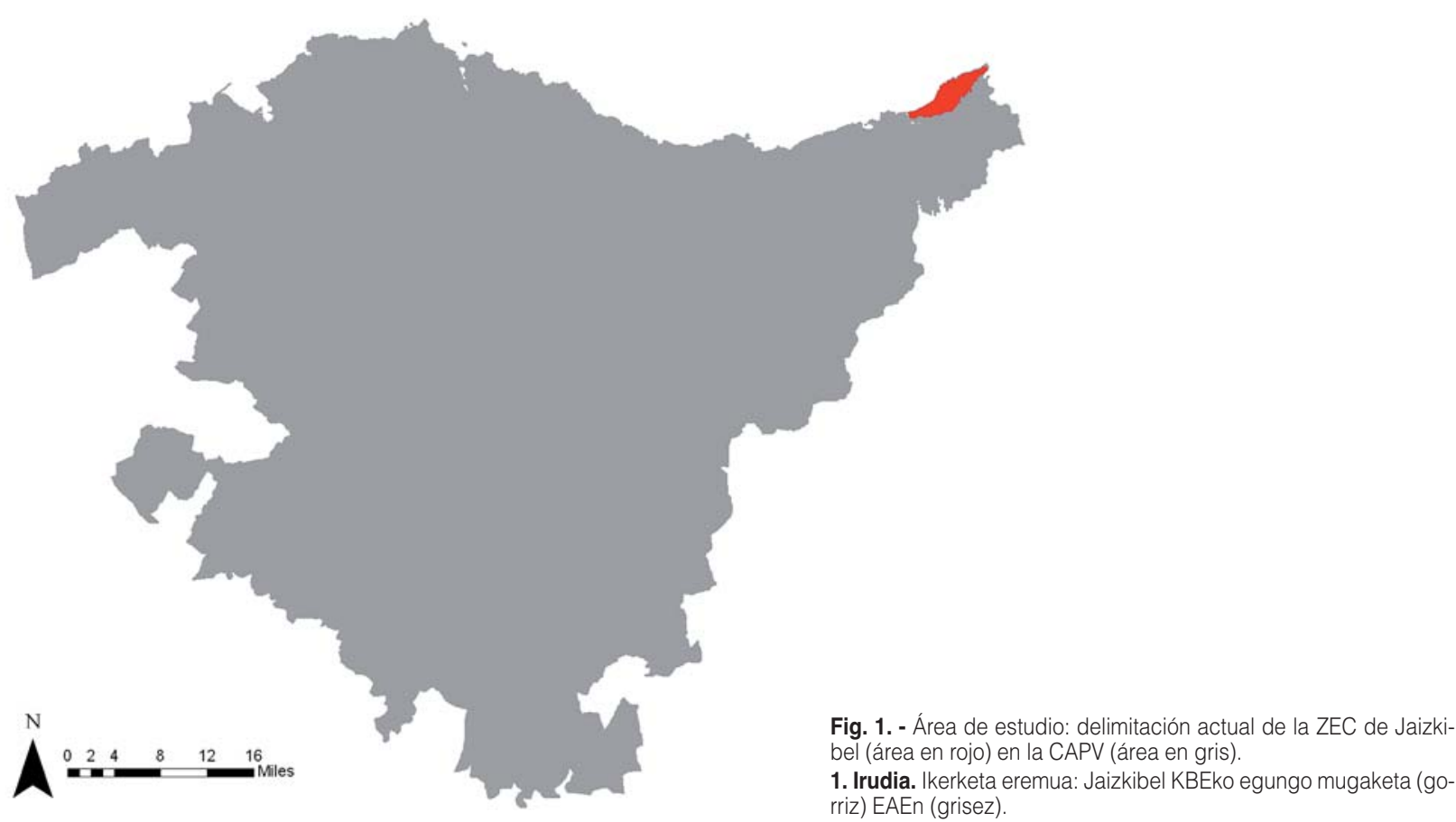


cial de la CAPV (Gobierno Vasco, 2003) y el mapa de vegetación potencial (Gobierno Vasco, 2006). De ese modo se calcularon datos de interés como el porcentaje que ocupan los hábitats naturales y seminaturales de Jaizkibel en relación con la superficie de los hábitats en toda la CAPV o en la Red Natura 2000 (RN2000) de toda la CAPV. Este último dato es orientativo ya que con el proceso de designación de las ZECs de la CAPV en curso están variando, aunque no de manera considerable. Del mismo modo, existe otro documento más actual de vegetación potencial, efectuado en 2011 en vez de 2006. No obstante se piensa que los datos mostrados sobre la superficie real de los hábitats actuales respecto a la potencial no varíen mucho utilizando uno u otro mapa.

\section{Listado y revisión de flora amenazada}

Para elaborar el listado de la flora amenazada presente en Jaizkibel, en primer lugar se efectuó una revisión bibliográfica detallada de las citas con la ayuda de la base de datos de la flora vascular ARAN-EH y el Herbario ARAN (Departamento de Botánica de la Sociedad de Ciencias Aranzadi). Posteriormente, se realizaron salidas de campo (periodo 2009-2013) para comprobar in situ las citas dudosas, antiguas o las que pudieran verse afectadas por algún factor externo.

Parte del trabajo de revisión de la flora amenazada se efectuó durante la redacción del Documento de Objetivos y Medidas para la designación de la Zona Especial de Conservación Jaizkibel (ES2120017) (OSTADAR, S.L. 2013). No obstante, con el paso del tiempo estos datos fueron completados con otras citas u observaciones recientes aportadas por colaboradores de este artículo (véase apartado Agradecimientos).

En el listado se incluyen las especies que aparecen en legislación vigente: Ios anexos II, IV y V de la Directiva Hábitat 92/43/CEE, la Lista Roja Española (Bañares et al., 2010), la Lista Roja de la flora vascular de la CAPV (IHOBE, 2010) y el Catálogo Vasco de Especies Amenazadas (BOVP, 2011)

\section{RESULTADOS}

\section{Hábitats naturales y seminaturales}

De las 2470 ha del área de estudio, 1743 ha (71\%) son hábitats naturales y seminaturales distribuidos en 25 tipos de hábitats EUNIS (Tabla 1). Una superficie de 942 ha (38\% del área de estudio) está ocupada por 12 hábitats de interés comunitario de los cuales 5 se consideran prioritarios. Un hábitat se considera prioritario cuando está amenazado de desaparición y su conservación supone una especial responsabilidad para la Unión Europea.

\section{Mapa de vegetación y otros usos}

El mapa de vegetación actual (Fig. 2) difiere mucho de la potencial, es decir del mapa de vegetación climácica que presentaría Jaizkibel sin la intervención humana formado por marojales (89\%), robledales (8\%), acantila-

\begin{tabular}{|c|c|c|c|c|c|}
\hline Nombre de hábitats EUNIS & CD & CE & NR & $\mathbf{S}$ & \%ZEC \\
\hline Acantilados y rocas costeras sin vegetación & & B3.23 & 4 & 53 & 2 \\
\hline Repisas y acantilados costeros con angiospermas halófilas & 1230 & B3.31 & 21 & 49 & 2 \\
\hline Láminas de agua de estuarios-rías, sin vegetación vascular & & $\mathrm{C} 2.4$ & 1 & $<1$ & $<1$ \\
\hline Trampales acidófilos-esfagnales & 7140 & D2.3 & 25 & 5 & $<1$ \\
\hline Trampales de Cladium mariscus & $7210^{*}$ & D5.24 & 1 & $<1$ & $<1$ \\
\hline Lastonares y pastos del Mesobromion & 6210 & E1.26 & 4 & 6 & $<1$ \\
\hline Praderas silicicolas de Deschampsia flexuosa & $6230^{*}$ & E1.73 & 17 & 72 & 3 \\
\hline Prados pastados y pastos no manipulados & & E2.11 & 24 & 145 & 6 \\
\hline Prados de siega atlánticos, no pastoreados & 6510 & E2.21 & 28 & 56 & 2 \\
\hline Prados-juncales basófilos atlánticos & & E3.41 & 1 & $<1$ & $<1$ \\
\hline Prados húmedos acidófilos dominados por Molinia & 6410 & E3.51 & 16 & 258 & 10 \\
\hline Helechales atlánticos y subatlánticos, colinos & & $\mathrm{E} 5.31(\mathrm{X})$ & 12 & 29 & 1 \\
\hline Helechales atlánticos y subatlánticos, montanos & & E5.31(Y) & 1 & 4 & $<1$ \\
\hline Zarzal acidófilo atlántico, con espinos (Rubus gr. glandulosus) & & F3.13 & 2 & 4 & $<1$ \\
\hline Argomal atlántico de Ulex europaeus & & $F 3.15(Y)$ & 43 & 398 & 16 \\
\hline Brezal húmedo con Erica ciliaris y E. tetralix & $4020^{*}$ & $F 4.12$ & 2 & 12 & $<1$ \\
\hline Brezal atlántico dominado por Ulex sp. & 4030 & $F 4.23(X)$ & 3 & 4 & $<1$ \\
\hline Brezal costero de Erica vagans & $4040^{*}$ & F4.231 & 34 & 359 & 15 \\
\hline Bortal o maquis alto termoatlántico & & $F 5.21(Y)$ & 1 & $<1$ & $<1$ \\
\hline Sauceda no riparia, de laderas rezumantes & & $\mathrm{F9} .2(\mathrm{Y})$ & 3 & 4 & $<1$ \\
\hline Seto de especies autóctonas & & FA.3 & 1 & $<1$ & $<1$ \\
\hline Aliseda ribereña eurosiberiana & $91 \mathrm{EO}^{*}$ & G1.21(Z) & 1 & 1 & $<1$ \\
\hline Marojal eurosiberiano & 9230 & G1.7B1 & 33 & 120 & 5 \\
\hline Bosque acidófilo dominado por Quercus robur & & G1.86 & 36 & 132 & 5 \\
\hline Bosques naturales jóvenes de frondosas & & G5.61 & 8 & 24 & 1 \\
\hline
\end{tabular}

Tabla 1. - Superficie ocupada por hábitats de interés en Jaizkibel. Los hábitats que presentan asterisco se consideran prioritarios en la Comunidad Europea. Abreviaturas: CD, Código de la Directiva Hábitats; CE, Código EUNIS NR, número de recintos; S, Superficie (en ha); \%ZEC, porcentaje de la superficie total de la ZEC Jaizkibel.

1. Taula. Interesezko habitatengatik okupatutako azalerak. Izartxoa daramaten habitatak lehentasunezkoak dira Europear Batasunean. Laburdurak: CD, Habitat Zuzentarauko kodea; CE, EUNIS kodea; NR, tesela kopurua; S azalera (ha-tan): \%ZEC, Jaizkibel KBEan habitatak okupatzen duen azalera portzentaia.

dos costeros $(<2 \%)$ y alisedas $(<2 \%)$ (OSTADAR, S.L. 2013). Salvo los acantilados costeros, el resto de los hábitats potenciales han sido sustituidos tanto por hábitats seminaturales como por artificiales en una gran parte de su extensión. En especial, los marojales y las alisedas han sido sustituidos en más del $90 \%$ de su superficie.

\section{Hábitats EUNIS naturales y seminaturales presentes en la actualidad en Jaizkibel}

De los diferentes tipos de hábitats EUNIS naturales y seminaturales que se pueden encontrar en la actualidad en Jaizkibel, se ha realizado el siguiente agrupamiento: formaciones boscosas, formaciones arbustivas, prados y pastos, acantilados costeros y trampales y esfagnales.

Las formaciones arbustivas (Fig. 3) son las que ocupan la mayor superficie de todas las formaciones con 815 ha $(33 \%)$. Además son las que más tipos de hábitats EUNIS presentan con los 10 hábitats que se muestran a continuación ordenados en función del área que ocupan: "Argomal atlántico de Ulex europaeus" 398 ha (16\%), "Brezal costero de Erica vagans" 359 ha (15\%), "Helechales atlánticos y subatlánticos, colinos" 29 ha (1\%), "Brezal húmedo con Erica ciliaris y E. tetralix" 12 ha $(<1 \%)$, "Zarzal acidófilo atlántico, con espinos (Rubus gr. glandulosus)" 4 ha (<1\%), "Sauceda no riparia, de laderas rezumantes" 4 ha $(<1 \%)$, "Brezal atlántico dominado por Ulex sp." 4 ha $(<1 \%)$, "Helechales atlánticos y subatlánticos, montanos) 4 


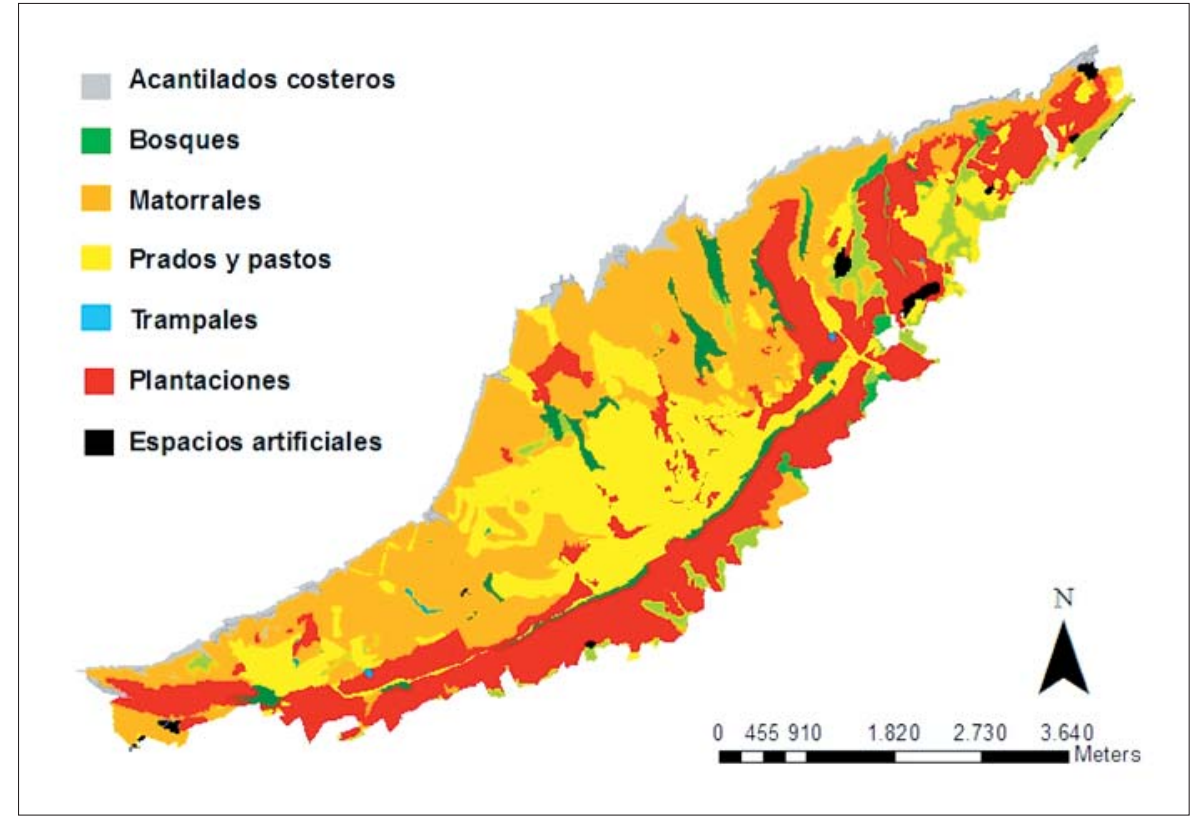

Fig. 2. - Mapa de vegetación y otros usos en la ZEC de Jaizkibel.

2. Irudia. Landarediaren eta beste erabileren mapa Jaizkibel KBEan.

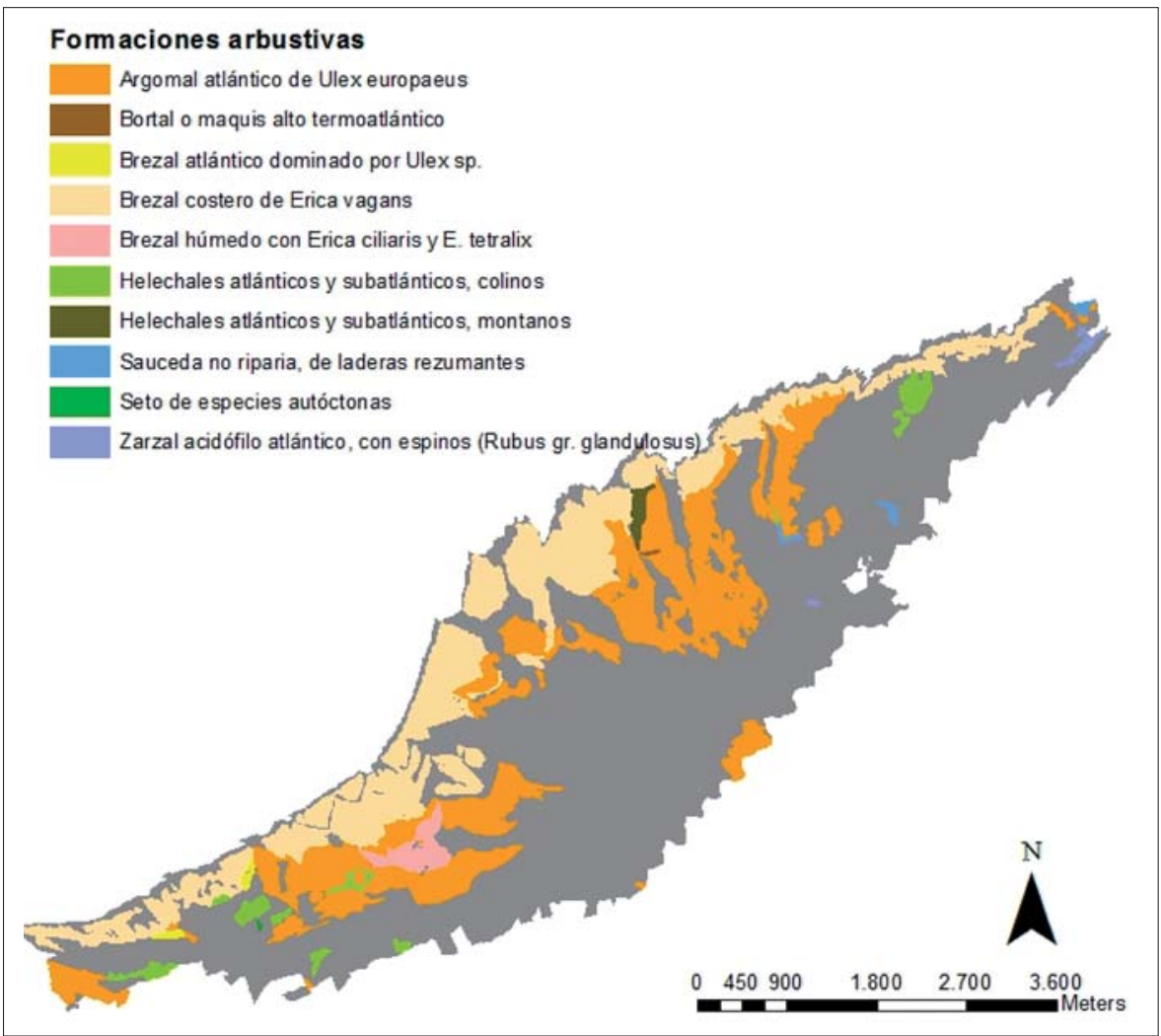

Fig. 3. - Formaciones arbustivas en la ZEC de Jaizkibel.

3. Irudia. Zuhaixka-erako formazioak Jaiz kibel KBEan.

ha $(<1 \%)$, "Bortal o maquis alto termoatlántico" $<1$ ha $(<1 \%)$ y "Seto de especies autóctonas" $<1$ ha $(<1 \%)$. Son hábitats de interés prioritario los dos primeros tipos de brezales citados.

En segundo lugar se encuentran los prados y pastos (Fig. 4), con una superficie de 538 ha (22\%) y 6 tipos de hábitats EUNIS: "Prados húmedos acidófilos dominados por Molinia" 258 ha (10\%), "Prados pastados y pastos no manipulados" 145 ha (6\%), "Praderas silicícolas de Deschampsia flexuosa" 72 ha (3\%)", "Prados de siega atlánticos, no pastoreados" 56 ha (2\%), "Lastonares y pastos del Mesobromiom" 6 ha $(<1 \%)$ y "Prados-juncales basófilos atlánticos" $<1$ ha $(<1 \%)$. Salvo el último hábitat y los "Prados pastados y pastos no manipulados", el resto son hábitats de interés comunitario, con las "Praderas silicícolas de Deschampsia flexuosa" como prioritarias. 


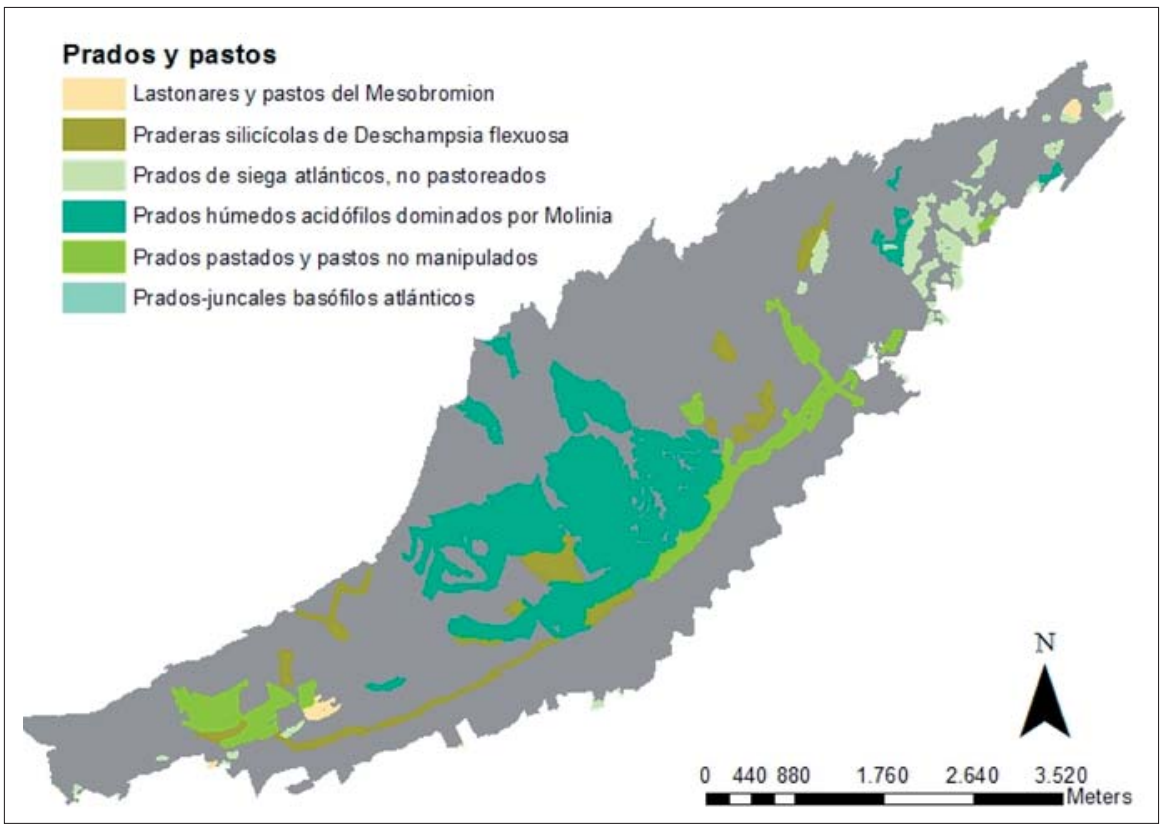

Fig. 4. - Prados y pastos en la ZEC de Jaizkibel. 4. Irudia. Larreak Jaizkibel KBEan.

A los prados y pastos les siguen las formaciones boscosas con 277 ha (12\%) (Fig. 5) repartidos en los siguientes hábitats EUNIS: "Marojal eurosiberiano" 120 ha (5\%), "Bosque acidófilo dominado por Quercus robur" 132 ha (5\%), "Bosques naturales de jóvenes frondosas" 24 ha ( $<1$ $\%)$ y "Aliseda ribereña eurosiberiana" 1 ha $(<1 \%)$. El "Marojal eurosiberiano" y la "Aliseda ribereña eurosiberiana" son hábitats de la Directiva Hábitat con la última de ellas además como prioritaria. Las plantaciones forestales con una ocupación de 645 ha (25\%) (Fig. 2) no se incluyen dentro de esta categoría.

En cuarto lugar se sitúan los acantilados costeros con una ocupación de 102 ha (4\%) (Fig. 6) y dos tipos de hábitats EUNIS: "Acantilados y rocas costeras sin vegetación"
53 ha (2\%) y "Repisas y acantilados costeros con angiospermas halófilas" 49 ha (2\%), el último de ellos prioritario.

Por último, se citan los trampales y esfagnales que ocupan una superficie de 6 ha (1\%) (Fig. 7) y los siguientes hábitats EUNIS: "Trampales acidófilos-esfagnales" 5 ha $(<1 \%)$ y "Trampales de Cladium mariscus" $<1$ ha $(<1 \%)$. Ambos son de interés comunitario y el último de ellos además prioritario.

\section{Superficie de los hábitats de la Directiva Hábitat de Jaiz- kibel en relación a la CAPV y la Red Natura 2000 vasca}

Son cinco los hábitats de interés comunitario que presentan una superficie mayor al $10 \%$ de su superficie total en la CAPV y en la RN2000 vasca. Los "Prados húmedos

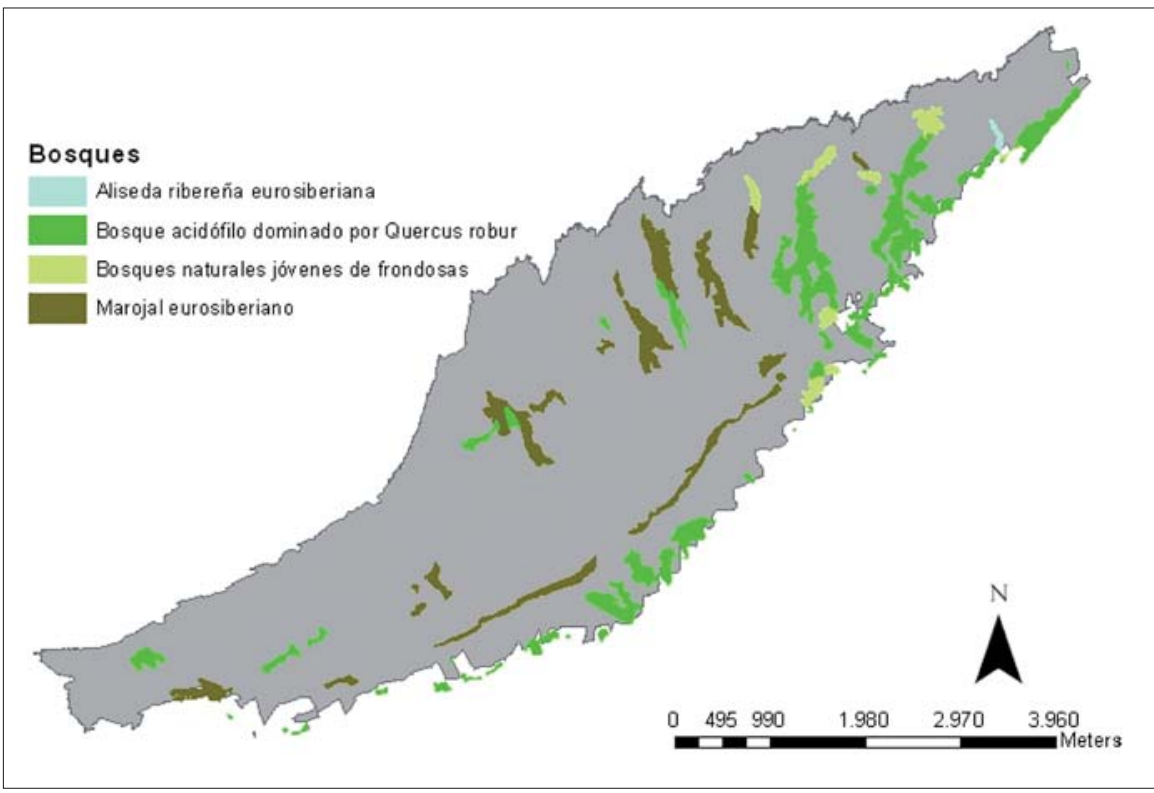

Fig. 5. - Formaciones boscosas en la ZEC de Jaizkibel.

5. Irudia. Baso-formazioak Jaizkibel KBEan 

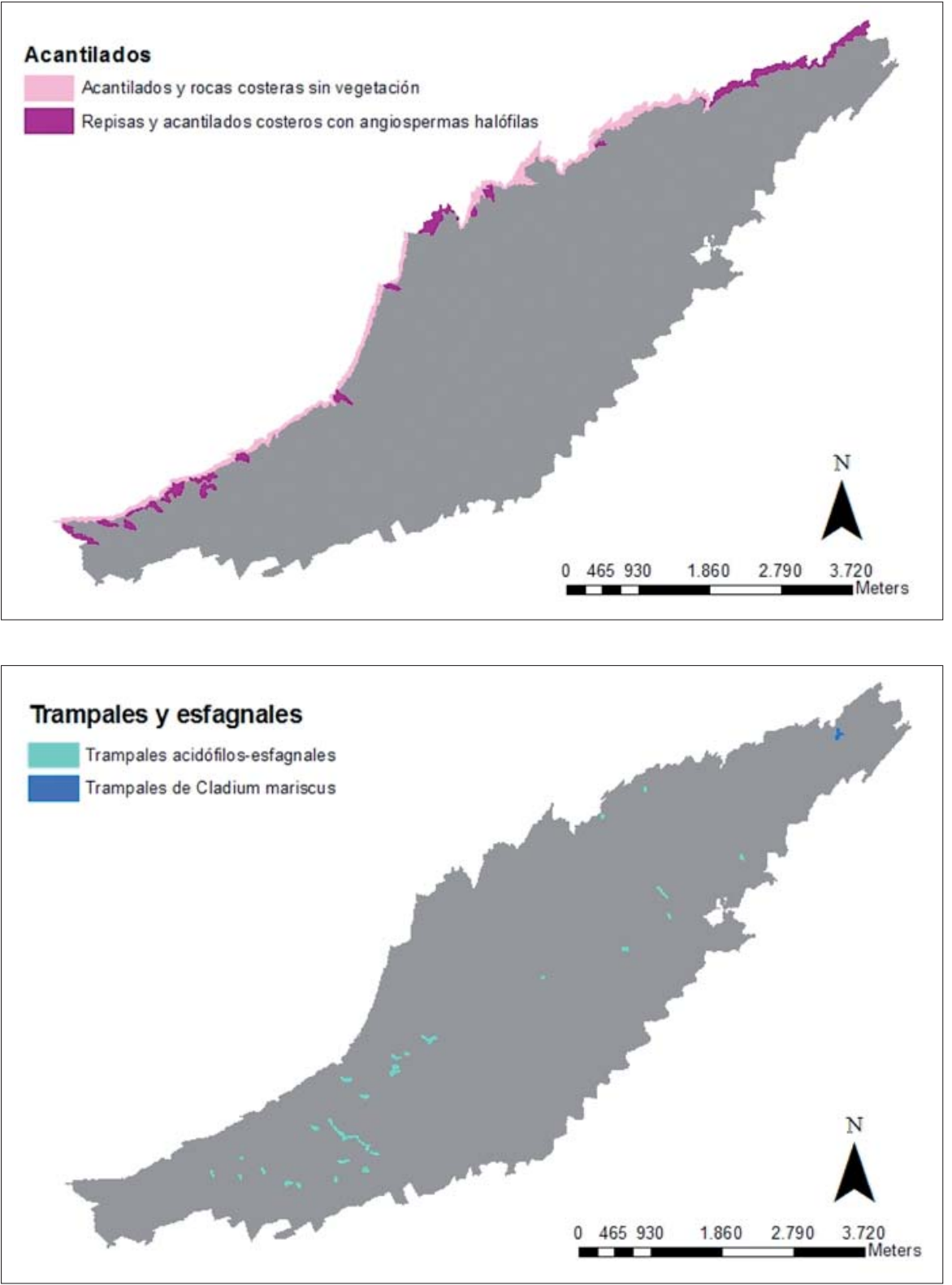

Fig. 6. - Acantilados costeros en la ZEC de Jaizkibel.

6. Irudia. Itsaslabarrak Jaizkibel KBEan.
Fig. 7. - Trampales y esfagnales en la ZEC de Jaizkibel.

7. Irudia. Hezegune eta esfagnadiak Jaizkibel KBEan. acidófilos dominados por Molinia" (Cód.UE 6410) de Jaizkibel suponen el $91 \%$ de la superficie de este hábitat en la CAPV y el 94\% de la superficie de este hábitat en la RN2000 vasca (Fig. 8). El resto de los hábitats de la CAPV y de la RN2000 vasca con mayor representación en Jaizkibel son los "Trampales de Cladium mariscus" (Cód.UE 7210*) con el $34 \%$ y el $43 \%$ respectivamente; el "Brezal costero de Erica vagans" (Cód. UE 4040*) con el 26\% y el 86\%; las "Repisas y acantilados costeros con angiospermas halófilas" (Cód.UE 1230 ) con el $19 \%$ y $59 \%$ y el "Brezal húmedo con Erica ciliaris y E. tetralix" (CódUE F4.12) con el 14\%.

\section{Especies}

Con los datos disponibles en las fuentes consultadas, en la actualidad la flora amenazada de Jaizkibel asciende hasta 14 especies (Tabla 2). Todas ellas se encuentran incluidas en el Catálogo Vasco de Especies Amenazadas (BOVP, 2011), 13 de ellas en la Lista Roja de la Flora Vascular de la CAPV (IHOBE, 2010), 3 en la Lista Roja Española (Bañares et al., 2010) y 5 en la Directiva Hábitat 92/43/CEE.

No se ha podido confirmar la presencia de otras cuatro especies: Carex hostiana DC. desde 1953, Dryopteris carthusiana (Vill) H.P. Fuchs. desde 1967, Radiola linoides Roth. desde 1985 y Rhynchospora fusca (L.) W.T. Aiton. desde 1941.

\section{DISCUSIÓN}

El marojal, que potencialmente ocuparía gran parte de la superficie de Jaizkibel, ha sido fuertemente sustituido por formaciones arbustivas, pastizales y plantaciones fo- 


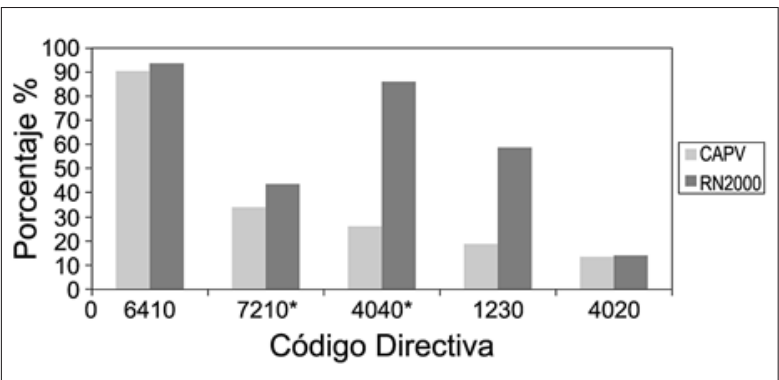

Fig. 8. - Hábitats de la Directiva Hábitat cuya presencia en Jaizkibel supone más del $10 \%$ de su superficie de la CAPV (gris claro) y de la Red Natura 2000 vasca (gris oscuro). Hábitats correspondientes a los códigos de la Directiva Hábitat: 6410- Prados húmedos acidófilos dominados por Molinia; 7210*- Trampales de Cladium mariscus; 4040*- Brezal costero de Erica vagans; 1230- Repisas y acantilados costeros con angiospermas halófilas; 4020*- Brezal húmedo con Erica ciliaris y E. tetralix. Se señalan los hábitats prioritarios con un asterisco.

8. Irudia. Habitat Zuzentarauko habitatak zeinak bere presentziak EAEan duen azaleraren \% 10a baino gehiago suposatzen duen (gris argiz) eta euskal Natura 2000 Sarean (gris ilunez). Habitat Zuentarauko kodeei dagozkien habitatak: 6410- Molinia caerulea-dun larre heze azidofiloak; 7210*- Cladium mariscus hezeguneak: 4040*- Kostako Erica vagans-en txilardia; 1230 Itsaslabarrak angiosperma halofilekin; 4020*- Erica ciliaris edota E. tetralixdun txilardi hezeak. Lehentasunezko habitatak izartxoarekin markatuta daude.

\begin{tabular}{|l|c|c|c|c|}
\hline Especies & CV & DA & LRE & LRC \\
\hline Armeria euscadiensis Donadille \& Vivant & VU & & NT & VU \\
\hline Carex hostiana DC* & EN & & VU & CR \\
\hline Drosera intermedia Hayne & EN & & & EN \\
\hline Dryopteris carthusiana (Vill) H.P. Fuchs* & VU & & & VU \\
\hline Hymenophyllum tunbrigense (L.) Sm. & VU & & VU & VU \\
\hline Ilex aquifolium L. & IE & & & LC \\
\hline Iris latifolia (Miller) Voss & VU & & & VU \\
\hline Juncus acutus L. & R & & & NT \\
\hline Koeleria albescens DC. & VU & & & VU \\
\hline Narcissus bulbocodium L. & R & V & & LC \\
\hline Pinguicula lusitanica L. & EN & & & NT \\
\hline Quercus suber L. & R & & & NT \\
\hline Radiola linoides Roth* & R & & & NT \\
\hline Rhynchospora fusca (L.) W.T. Aiton* & EN & & EN & EN \\
\hline Ruscus aculeatus L. & IE & V & & \\
\hline Spiranthes aestivalis (Poiret) L.C.M. Richard & VU & IV & & VU \\
\hline Thelypteris palustris Schott & EN & & & EN \\
\hline Trichomanes speciosum Willd. & VU & II & VU & VU \\
\hline Woodwardia radicans (L.) Sm. & VU & II & & VU \\
\hline
\end{tabular}

Tabla 2. - Inventario de la flora amenazada de Jaizkibel. II, IV y V: plantas presentes en los Anexos II, IV y V de la Directiva Hábitat respectivamente. Abreviaturas: CV, Catálogo Vasco de Flora Amenazada (2011); DA, Directiva Aves; LRE, Lista Roja de España (2010); LRC, Lista Roja de la CAPV (2010): VU: vulnerable; NT: casi amenazada; EN: en peligro; CR: en peligro crítico; IE: de interés especial; LC: preocupación menor; R: rara. * Especies sin confirmar su presencia en la actualidad.

2. Taula. Jaizkibeleko flora mehatxatuaren inbentarioa. II, IV eta V: Habitat Zuzentarauko II, IV eta V eranskinetan azaltzen diren landareak. Laburdurak: CV, Flora Mehatxatuaren Euskadiko Zerrenda (2011); DA, Hegaztien Zuzentaraua; LRE, Espainiko Zerrenda Gorria (2010); LRC, EAEko Zerrenda Gorria; VU: kaltebera; NT: ia zaurgarria; EN: galtzeko arriskuan; CR: galtzeko arrisku larrian; IE: interes berezikoa; LC: arriskurik gabea; R: bakana. * Egun, presentzia ziurtatu gabeko espezieak.

restales. Así, de poder ocupar aproximadamente el 89\% del lugar, actualmente presentan una superficie del $5 \%$. Las pocas manchas presentes en el macizo se encuentran fragmentadas y la mayoría son masas jóvenes que no han podido evolucionar por los sucesivos incendios que ha sufrido Jaizkibel durante años (OSTADAR, S.L. 2013). Las alisedas y los robledales también se han visto considerablemente sustituidos, del orden del 90\% para el pri- mer hábitat y del 38\% para el segundo. Los acantilados de Jaizkibel todavía muestran un estado semivirgen (OSTADAR, S.L. 2013) por lo que no se calcula sustitución frente a la potencial para estas formaciones. No obstante, la compleja orografía que presenta Jaizkibel hace necesaria una cartografía más detallada de los hábitats que se encuentran en las zonas de mayor verticalidad como los acantilados o los bosques de ribera. Esto supondría un aumento de la superficie real de estos hábitats que en las circunstancias actuales se encuentran infravalorados.

Como plantas amenazadas que se relacionan con las formaciones forestales y sus etapas sucesionales se encuentran Ruscus aculeatus L., Narcissus bulbocodium L., Dryopteris carthusiana (Vill) H.P. Fuchs., Ilex aquifolium L. y Quercus suber L. Salvo D. carthusiana, no observada en Jaizkibel desde 1967 (Braun-Blanquet, 1967), se trata de especies que no presentan un riesgo elevado de desaparición a nivel de la CAPV pero aún así su presencia en Jaizkibel es interesante por varios motivos: $Q$. suber es una especie de distribución mediterránea con algunas poblaciones en la región atlántica; I. aquifolium y $R$. aculeatus son plantas que han sufrido antiguamente una fuerte recolección para el uso ornamental; y $N$. bulbocodium y $R$. aculeatus son especies incluidas en el Anexo V de la Directiva, cuya recogida en la naturaleza y cuya explotación pueden ser objeto de medidas de gestión.

Jaizkibel es un lugar interesante a nivel regional para la conservación de los "Prados húmedos acidófilos dominados por Molinia" ya que, como se ha dicho, estos prados de interés comunitario que ocupan 253 ha del espacio protegido representan más del $90 \%$ de su superficie de toda la CAPV y de la RN2000 vasca. Además, se ha propuesto Jaizkibel como lugar clave de la CAPV dentro del protocolo estatal para establecer un sistema de vigilancia global del estado de conservación del hábitat en cuestión (Marinas et al., 2009). Estos prados ocupan suelos húmedos por la existencia de un nivel freático alto aunque fluctuante y las principales amenazas para el hábitat a nive estatal son la desecación de los suelos y la fragmentación del hábitat (Marinas et al., 2009). Asociado a herbazales húmedos se encuentra Radiola linoides Roth. citada en 1967 por Braun-Blanquet, J. y observada por última vez en 1985 (Catalán et al., 1985).

Asimismo, Jaizkibel es interesante a nivel de la CAPV por los "Trampales de Cladium mariscus" con una mancha cartografiada, aunque recientemente se han observado más enclaves de $C$. mariscus (comentario personal de Yves Meyer, EKOS Estudios Ambientales). La presencia de C. mariscus en otros lugares hace valorar la posibilidad de tener que volver a cartografiar esos enclaves como trampales, ya que la identificación de este tipo de hábitat exige, además de la presencia de la especie característica C. mariscus, la presencia de otras especies características de la comunidad Caricion davallianae y la existencia de un sustrato turboso o suelos ricos en materia orgánica. Además, es un hábitat difícil de determinar con precisión apareciendo frecuentemente ocupando áreas dispersas no cartografiables, intercaladas con otros hábitats de humedales (García-Rodeja et al., 2009). En la formación de 
C. mariscus se encuentra una población del helecho circumboreal amenazado llamado Thelypteris palustris Schott, que vive en lugares frescos y encharcados (Zendoia et al., 2007; confirmada su presencia en primavera del 2013 por Mari Azpiroz y Anaïs Mitxelena).

En los trampales y las zonas encharcables se encuentra una muestra importante de la flora amenazada de Jaizkibel. Además de T. palustris mencionada en el párrafo anterior, destaca la orquídea ligada a medios turbosos o paraturbosos Ilamada Spiranthes aestivalis (Poiret) L.C.M. Richard, de la que en 2008 se observaron 23 reproductores en un trampal (Garmendia et al., 2008). En 2013 se ha confirmado el núcleo (Garmendia et al., 2014) pero se da por desaparecido el núcleo de otro trampal citado por Catalán y Aizpuru (1985) ya que no se han encontrado durante las prospecciones de 2009 ni en años anteriores (Zendoia et al., 2003; Zendoia et al., 2006; Heras et al., 2009). Otra planta amenazada ligada a estos hábitats es Carex hostiana DC., citada en el pasado (Allorge \& Allorge, 1941; Guinea 1953) pero cuya presencia no se ha confirmado recientemente. Esta especie habita en pastizales encharcados, orillas de manantiales y brezales húmedos. Asimismo, ligados a estos ambientes se citan dos plantas carnívoras: Drosera intermendia Hayne y Pinguicula lusitanica L. D. intermedia (Zendoia et al., 2003) está ligada a turberas y humedales ácidos y $P$. Iusitanica ocupa a menudo pequeños taludes arcillosos húmedos pero no inundables. Esta última especie ha sido citada en diez esfagnales de Jaizkibel (Zendoia et al., 2007) aunque se piensa que, por la cantidad de observaciones realizadas actualmente por Yves Meyer, de EKOS Estudios Ambientales, esta cifra podría ser mayor. Por último, ligado a estos ambientes existe una cita de Rhynchospora fusca (L.) W.T. Aiton de Allorge et al., (1941) dada por verosímil y que no ha sido observada desde entonces tal como se indica en Aseginolaza et al., (1984).

Del mismo modo, el macizo debe considerarse clave por los brezales costeros y los acantilados costeros. Los brezales costeros (Cod.UE.4040*) son exclusivos de la región atlántica y en la Península Ibérica se distribuyen desde Portugal hasta la CAPV. A nivel estatal se trata de una formación en disminución por la influencia humana y con perspectivas inadecuadas (Ojeda, 2009). Estos brezales, que están desprovistos de cubierta arbórea, necesitan de los vientos marinos y están sometidos a la brisa marina que aporta agua salada y partículas de sal. Así mismo, para su conservación sería necesario un mecanismo de control de Baccharis halimifolia L., planta alóctona invasora de porte arbustivo alto en rápida invasión de la costa de Jaizkibel. Este problema también afecta a otros hábitats cercanos a la costa como es la formación de C. mariscus (Azpiroz et al., 2012).

En primera línea frente al mar se encuentran los acantilados costeros. Los llamados "Repisas y acantilados costeros con angiospermas halófilas" (Cod.UE.1230) presentan fuertes pendientes, suelo escaso y soportan fuertes vientos marinos. Se distribuyen por las costas peninsulares del océano Atlántico (Lopez-Bedoya \& Pérez-Alberti, 2009). Aunque los hábitats costeros son los más afectados a nivel de la CAPV por la ocupación del suelo para creación de viviendas, infraestructuras y equipamientos, los acantilados y brezales costeros presentan estados más favorables de conservación que las marismas y los arenales (IHOBE, 2012). La aparición de plantas que aparecen en hábitats alterados por la acción del ser humano, la presencia de especies invasoras (como Stenotaphrum secundatum (Walter) Kuntze presente en Jaizkibel) y la creación de senderos (por ejemplo el concurrido sendero Talaia que recorre la costa vasca) son factores antrópicos que repercuten en los acantilados. Aunque al principio podría parecer que la creación de senderos tiene escasa relevancia en la estabilidad de los acantilados, estudios realizados en la Bretaña Francesa demuestran lo contrario (López-Bedoya \& Pérez-Alberti, 2009)

Como especies ligadas a ambientes del litoral y que crecen en la línea costera, en los acantilados costeros y brezales costeros destaca especialmente la endémica Armeria euscadiensis Donadille \& Vivant muy abundante en Jaizkibel, además de otras también catalogadas como Iris latifolia (Miller) Voss vista en 2012 al menos dos lugares según Yves Meyer, de EKOS Estudios Ambientales, y censadas en 2013 contabilizando hasta 14 y 150-200 individuos en cada núcleo (Garmendia et al., 2014), y Juncus acutus L. (OSTADAR S.L. 2013).

En ambientes nemorales donde la vegetación de ribera como las alisedas (Cod.UE.91E0*) o las cascadas proporcionan una humedad relativa alta encontramos tres helechos paleotropicales. Por un lado se encuentra Woodwardia radicans (L.) Sm. con un núcleo en el arroyo de Martitxene (confirmada por Iñaki Sanz e Ibai Olariaga en verano de 2012). El segundo helecho es Hymenophyllum tunbrigense (L.) Sm. con dos núcleos en el arroyo Justiz y otro en la regata de Gaztarrotz (Catalán \& Aizpuru, 1985; confirmada en invierno de 2012 por Iñaki Sanz, Iñaki Aizpuru e Yves Meyer). El último helecho ligado a estos ambientes es Trichomanes speciosum Willd. con 2 localidades conocidas en 2009 y muchas más localidades conocidas en la actualidad gracias al trabajo realizado por Sanz-Azkue et al. (en prensa). Estas poblaciones se ven amenazadas por modificaciones del ciclo hidrológico y las prácticas forestales, donde normalmente no solamente se tala la vegetación existente en el arroyo, sino que quedan totalmente cegados por la acumulación de ramas arrojadas tras las cortas (IHOBE, 2010). Así mismo, el uso del fuego y los desbroces del matorral han hecho desaparecer la vegetación de ribera de los arroyos de la zona oriental y, a pesar de que la parte occidental esté más arbolada en la actualidad, su estado de conservación no es favorable (OSTADAR, S.L., 2013).

Desde que se inició el proceso de designación de la Zona Especial de Conservación Jaizkibel (ES2120017) en 2009, el interés sobre los valores naturales y culturales del macizo aumentó en la población en general. Así, Jaizkibel ha sufrido un aumento de visitas en los últimos años encontrándose nuevas localidades de la flora amenazada inventariada en este trabajo. Por ello, sería necesario realizar un trabajo conjunto de actualización de los 
mapas de distribución de al menos I. latifolia, A. euscadiensis y P. Iusitanica.

Se concluye que una cartografía actualizada de la flora amenazada de Jaizkibel, junto con la cartografía de hábitats del lugar, son las mejores herramientas para poder gestionar los hábitats y las especies y hacer frente a las amenazas que hacen peligrar su conservación en dicho macizo.

\section{AGRADECIMIENTOS}

Nuestro agradecimiento a Iñaki Sanz-Azkue, Ibai Olariaga, Yves Meyer, Iñaki Aizpuru, Joseba Garmendia, Mari Azpiroz por la información aportada. A Santiago García y la empresa OSTADAR S.L. por habernos brindado la oportunidad de profundizar el conocimiento sobre Jaizkibel.

\section{BIBLIOGRAFÍA}

Allorge, V., Allorge, P. 1941. Plantes rares ou intéressantes du Nord-Ouest de l'Espagne, principalement du Pays Basque. Bull. Soc. Bot. France. 88: 291-357.

Azpiroz, M., Mitxelena, A., Garin-Barrio, I., Zulaika, I., Mendiola, I., Oreja, L., Garmendia, J. 2012. Erradicación de la especie invasora Baccharis halimifolia y restauración de formaciones de Cladium mariscus y brezales costeros de Erica vagans en Aizporaundi (Jaizkibel). Sociedad de Ciencias Aranzadi para Hondarribiko Udala.

Bañares, A., Blanca, G., Guemes, J., Moreno, J.C., Ortiz, S. (Ed.). 2010. Atlas y Libro Rojo de la Flora Vascular Amenazada de España. Adenda 2010. Dirección General de Medio Natural y Política Forestal (Ministerio de Medio Ambiente, y Medio Rural y Marino)-Sociedad Española de Biología de la Conservación de Plantas. Madrid.

BOPV. 2011. ORDEN de enero de 2011, de la Consejera de Medio Ambiente, Planificación Territorial, Agricultura y Pesca, por la que se modifica el Catálogo Vasco de Especies Amenazadas de la Fauna y Flora Silvestre y Marina, y se aprueba el texto único. CAPV-miércoles 23 de febrero de 2011 № 37.

Braun-Blanquet, J. 1967. Vegetationsskizzen aus dem Basquenland mit Ausblicken auf das Weitere Ibero-Atlantikum. I. Vegetatio. 14: 1-126.

Catalán, P., Aizpuru, I. 1985. Aportación al catálogo florístico de la cuenca del Bidasoa (Guipúzcoa y Navarra). Munibe, Cienc. Nat. 37: 17-86.

DOCE. 1992. Directiva 92/43/CEE del consejo de 21 de Mayo de 1992 relativa a la Conservación de los Hábitats Naturales y de la Fauna y Flora Silvestre. Diario Oficial de las Comunidades Europeas, 22/07/1992.

García-Rodeja, E., Fraga, M. I., Fidalgo, C., González, J. A. 2009. 7210 Areas pantanosas calcáreas con Cladium Mariscus y especies de Caricion Davallianae $\left(^{*}\right)$. En: Bases ecológicas preliminares para la conservación de los tipos de hábitat de interés comunitario en España. VV.AA. (Ed.): Ministerio de Medio Ambiente, y Medio Rural y Marino.

Garmendia, J., Oreja, L., Azpiroz, M., Uriarte, L. 2008. Trabajos para la conservación de la flora amenazada de Gipuzkoa (campaña de 2007). Informe realizado para la Diputación de Gipuzkoa. Sociedad de Ciencias Aranzadi. Donostia-San Sebastián.

Garmendia, J., Azpiroz, M., García, Y., Uriarte, L. 2014. Gipuzkoako landare espezie mehatxatuen jarraipen-lanak. Informe inédito realizado por la Sociedad de Ciencia Aranzadi para la Diputación Foral de Gipuzkoa.
Gobierno Vasco. 2003. Mapa de los Lugares de Importancia Comunitaria (LIC) y Zonas Especiales de Conservación (ZEC) Escala 1:25.000. Gobierno Vasco.

Gobierno Vasco. 2006. Mapa de series de vegetación (vegetación potencial) de la Comunidad Autónoma del País Vasco a escala 1:50.000. Departamento de Medio Ambiente, Planificación Territorial, Agricultura y Pesca.

Gobierno Vasco. 2007. Cartografía de hábitats, vegetación ac tual y usos del suelo de I Comunidad Autónoma del País Vasco. Escala 1:10.000. Departamento de Medio Ambiente, Planificación Territorial, Agricultura y Pesca.

Gobierno Vasco. 2009. Ortofoto $200925 \mathrm{~cm}$ de la Comunidad Autónoma del País Vasco. Servicio de Cartografía, Departamento de Medio Ambiente, Planificación Territorial, Agricultura y Pesca.

Guinea, E. 1953. Geografía botánica de Santander. Santander

Heras, P., Infante, M., Zendoia, I. 2009. Trabajos de gestión de Turberas y Esfagnales. II Seguimiento de los enclaves de Jaizkibel, campaña 2007. Diputación Foral de Gipuzkoa. Informe técnico.

IHOBE. 2010. Lista roja de la flora vascular de la CAPV Gobierno Vasco. Departamento de Medio Ambiente, Planificación Territorial, Agricultura y Pesca.

IHOBE. 25 de enero de 2012. Evaluación de los hábitats terrestres de interés comunitario en la Comunidad Autónoma del País Vasco 2007-2012. Documento inédito.

López-Bedoya, J. L., Pérez-Alberti, A. 2009. 1230 Acantilados con vegetación de las costas atlánticas y bálticas. En: Bases ecológicas preliminares para la conservación de los tipos de hábitat de interés comunitario en España. VV.AA. (Ed.): 144 Ministerio de Medio Ambiente, y Medio Rural y Marino.

Marinas, A., Gómez, D., García-González, R. 2009. 6410 Prados-juncales con Molinia caerulea sobre suelos húmedos gran parte del año. En: Bases ecológicas preliminares para la conservación de los tipos de hábitat de interés comunitario en España. VV.AA. (Ed.): 54. Ministerio de Medio Ambiente, y Medio Rural y Marino.

Ojeda, F. 2009. 4040 Brezales costeros con Erica vagans (*). En: Bases ecológicas preliminares para la conservación de los tipos de hábitat de interés comunitario en España. VV. AA (Ed.). Ministerio de Medio Ambiente, y Medio Rural y Marino. Madrid.

OSTADAR, S.L. 2013. Documento de Objetivos y Medidas para la designación de la Zona Especial de Conservación Jaizkibel (ES2120017). OSTADAR, S.L. para la Dirección de Biodiversidad y Participación del Departamento de Medio Ambiente, Planificación Territorial, Agricultura y Pesca. Gobierno Vasco. Documento para aprobación definitiva.

Sanz-Azkue, I., Olariaga, I., Felipe, A En prensa Trichomanes speciosum en Jaizkibel: nuevas poblaciones con alta representación de su fase gametofítica y modelización de su distribución. Sociedad de Ciencias Aranzadi.

Zendoia, I., Arbelaitz, E., Azpiroz, M., Garmendia, J., Oreja, L., Tamayo, I., Urkizu, A., Heras, P., Infante, M. 2006. Europar Habitat Arteztarauak babesten dituen zohikaztegia eta esfaganadien kudeaketarako lanak. Diputación Foral de Gipuzkoa. Informe técnico.

Zendoia, I., Gartzia, M., Aldezabal, A., Aizpuru, I. 2003. Gipuzkoako kostaldeko esfagnadien kartografia, karakterizazioa eta kontserbazio-egoera. Munibe, Cienc. Nat. 54: 5-20.

Zendoia, I., Oreja L., Garmendia J., Azpiroz M., Arbelaitz E., Urkizu A., Tamayo I., Aizpuru, I. 2007 Cladium mariscus formazioen kartografia, karakterizazioa eta kontserbazio-egoera. Eusko Jaurlaritza. Informe inédito. 